\title{
Assessing digital micromirror devices for speckle noise control in digital holography
}

\section{Evaluación de dispositivos digitales de microespejos para el control del ruido speckle en holografía digital}

\author{
Jefry Gaviria-Mesa ${ }^{1}$, Diego Hincapie-Zuluaga ${ }^{1,2}$, Jorge Garcia-Sucerquia ${ }^{2}$, Nelson Correa- \\ Rojas $^{1}$, Jorge Herrera-Ramírez ${ }^{1}$ \\ 1. Facultad de Ingeniería, Instituto Tecnológico Metropolitano, Calle 73 No 76A-354, \\ Medellin 050034, Colombia \\ 2. Facultad de Ciencias, Universidad Nacional de Colombia, A.A. 3840, Sede Medellin, Colombia \\ ${ }^{(*)}$ E-mail: jorgeherrerar@itm.edu.co
}

Received: 09/12/2016 Accepted: 31/07/2017

DOI: 10.7149/OPA.50.3.49069

\begin{abstract}
:
Speckle noise is a common issue in coherent imaging techniques. As one of these techniques, digital holography (DH) is affected by this problem. Although several attempts have been made to overcome speckle noise in the numerical reconstruction of digitally recorded holograms, there still exists the need for fast and effective methods to get rid of speckle and improve image quality. We assess the suitability of a digital micromirror device (DMD) as modulator of the object illumination in a digital holographic setup, where the DMD acts generating speckle-like patterns that are projected onto the object. These patterns change the object beam in a random way. Several holograms, each with a different pattern in the object illumination, are recorded and numerically reconstructed. The image with reduced speckle noise is the result of a superposition in an intensity basis of various such reconstructions. The feasibility of the method is validated by experimental results.
\end{abstract}

Key words: Digital holography; Speckle; Digital Micromirror Device

\section{RESUMEN:}

El ruido speckle es un problema inherente a las técnicas de formación de imágenes con luz coherente. Ya que la holografía digital es una de estas técnicas, también se ve afectada por este problema. Aunque se han hecho varios intentos por eliminar el ruido speckle en la reconstrucción numérica de hologramas registrados digitalmente, todavía existe la necesidad de métodos rápidos y efectivos para eliminar el speckle y mejorar la calidad de las imágenes reconstruidas. Nosotros evaluamos la conveniencia del uso de un dispositivo digital de microespejos (DMD) como modulador de la iluminación del objeto en un montaje holográfico digital. El DMD actúa generando patrones aleatorios tipo speckle que son proyectados sobre el objeto. Estos patrones cambian el haz objeto de una manera aleatoria y después interfiere formando el holograma. Se registran varios hologramas, cada uno con un patrón diferente en la iluminación del objeto, y se reconstruyen numéricamente. La imagen final con el ruido speckle reducido es el resultado de la superposición en intensidades de varias de estas reconstrucciones. La viabilidad del método es validada mediante resultados experimentales.

Palabras clave: Holografía digital, Speckle, Digital Micromirror Device. 


\section{REFERENCES AND LINKS}

[1] J. W. Goodman and R. W. Lawrence, "Digital image formation from electronically detected holograms", Appl. Phys. Lett. 11, 77-79 (1967).

https://doi.org/10.1063/1.1755043

[2] E. Tajahuerce and B. Javidi, "Encrypting three-dimensional information with digital holography", Appl. Opt. 39, 6595 (2000). https://doi.org/10.1364/A0.39.006595

[3] L. Wilson and R. Zhang, "3D Localization of weak scatterers in digital holographic microscopy using Rayleigh-Sommerfeld back-propagation", Opt. Express 20, 16735 (2012). https://doi.org/10.1364/OE.20.016735

[4] M.-K. Kim, "Applications of Digital Holography in Biomedical Microscopy", J. Opt. Soc. Korea 14, 7789 (2010). https://doi.org/10.3807/JOSK.2010.14.2.077

[5] S. Teeranutranont and K. Yoshimori, "Digital holographic three-dimensional imaging spectrometry.", Appl. Opt. 52, A388-96 (2013). https://doi.org/10.1364/A0.52.00A388

[6] Y. Lu, Y. Liu, and T. K. Lau, "Simple, portable, and low-cost microscope based on off-axis digital holography using two spherical waves.", Opt. Lett. 39, 4549-52 (2014). https://doi.org/10.1364/OL.39.004549

[7] P. Ferraro, G. Coppola, S. De Nicola, A. Finizio, S. Grilli, M. Iodice, C. Magro, and G. Pierattini, "Digital holography for characterization and testing of MEMS structures", IEEE/LEOS Int. Conf. Opt. MEMs 125-126 (2002). https://doi.org/10.1109/OMEMS.2002.1031475

[8] M. H. Jericho, H. J. Kreuzer, M. Kanka, and R. Riesenberg, "Quantitative phase and refractive index measurements with point-source digital in-line holographic microscopy.", Appl. Opt. 51, 1503-15 (2012). https://doi.org/10.1364/A0.51.001503

[9] Z. Frentz, S. Kuehn, D. Hekstra, and S. Leibler, "Microbial population dynamics by digital in-line holographic microscopy.", Rev. Sci. Instrum. 81, 84301 (2010). https://doi.org/10.1063/1.3473937

[10] E. Cuche, F. Bevilacqua, and C. Depeursinge, "Digital holography for quantitative phase-contrast imaging", Opt. Lett. 24, 291 (1999). https://doi.org/10.1364/OL.24.000291

[11] J. Jung, K. Kim, H. Yu, K. Lee, S. Lee, S. Nahm, H. Park, and Y. Park, "Biomedical applications of holographic microspectroscopy [Invited]", Appl. Opt. 53, G111 (2014). https://doi.org/10.1364/A0.53.00G111

[12] J. W. Goodman, "Some fundamental properties of speckle", J. Opt. Soc. Am. 66, 1145 (1976). https://doi.org/10.1364/JOSA.66.001145

[13] Y. Imai and Y. Ohtsuka, "Laser speckle reduction by ultrasonic modulation", Opt. Commun. 27, 18-22 (1978). https://doi.org/10.1016/0030-4018(78)90165-7

[14] C. Liu, Y. Chang, K.-W. Lin, and P. Lin, "Speckle reduction in laser imaging applications using rotating magneto-optical disk", J. Opt. Soc. Am. A 31, 16 (2014). https://doi.org/10.1364/JOSAA.31.000016

[15] B. Redding, G. Allen, E. R. Dufresne, and H. Cao, "Low-loss high-speed speckle reduction using a colloidal dispersion.", Appl. Opt. 52, 1168-72 (2013). https://doi.org/10.1364/A0.52.001168

[16] T.-T.-K. Tran, Ø. Svensen, X. Chen, and M. Nadeem Akram, "Speckle reduction in laser projection displays through angle and wavelength diversity", Appl. Opt. 55, 1267 (2016). https://doi.org/10.1364/A0.55.001267

[17] T. Tschudi, "Speckle reduction in laser projections with ultrasonic waves", Opt. Eng. 39, 1659 (2000). https://doi.org/10.1117/1.602543

[18] J. Maycock, B. M. Hennelly, J. B. McDonald, Y. Frauel, A. Castro, B. Javidi, and T. J. Naughton, "Reduction of speckle in digital holography by discrete Fourier filtering", J. Opt. Soc. Am. A 24, 1617 (2007). https://doi.org/10.1364/JOSAA.24.001617

[19] D. Hincapie, J. Herrera-Ramírez, and J. Garcia-Sucerquia, "Single-shot speckle reduction in numerical 
reconstruction of digitally recorded holograms", Opt. Lett. 40, 1623 (2015) . https://doi.org/10.1364/OL.40.001623

[20] T. Fukuoka, Y. Mori, and T. Nomura, "Speckle Reduction by Spatial-Domain Mask in Digital Holography", J. Disp. Technol. 1-1 (2015).

[21] P. Memmolo, I. Esnaola, A. Finizio, M. Paturzo, P. Ferraro, and A. M. Tulino, "SPADEDH: a sparsitybased denoising method of digital holograms without knowing the noise statistics", Opt. Express 20, 17250 (2012). https://doi.org/10.1364/OE.20.017250

[22] V. Bianco, M. Paturzo, P. Memmolo, A. Finizio, P. Ferraro, and B. Javidi, "Random resampling masks: a non-Bayesian one-shot strategy for noise reduction in digital holography.", Opt. Lett. 38, 619-21 (2013). https://doi.org/10.1364/OL.38.000619

[23] A. Uzan, Y. Rivenson, and A. Stern, "Speckle denoising in digital holography by nonlocal means filtering", Appl. Opt. 52, A195 (2013). https://doi.org/10.1364/A0.52.00A195

[24] A. Sharma, G. Sheoran, Z. a. Jaffery, and Moinuddin, "Improvement of signal-to-noise ratio in digital holography using wavelet transform", Opt. Lasers Eng. 46, 42-47 (2008). https://doi.org/10.1016/j.optlaseng.2007.07.004

[25] J. Garcia-Sucerquia, J. Herrera-Ramirez, and D. Velasquez-Prieto, "Reduction of speckle noise in digital holography by using digital image processing", Opt. - Int. J. Light Electron Opt. 116, 44-48 (2005). https://doi.org/10.1016/j.ijleo.2004.12.004

[26] J. H. Massig, "Digital off-axis holography with a synthetic aperture", Opt. Lett. 27, 2179 (2002). https://doi.org/10.1364/OL.27.002179

[27] J. Bühl, H. Babovsky, A. Kiessling, and R. Kowarschik, "Digital synthesis of multiple off-axis holograms with overlapping Fourier spectra", Opt. Commun. 283, 3631-3638 (2010). https://doi.org/10.1016/j.optcom.2010.05.038

[28] X. Cai and H. Wang, "The influence of hologram aperture on speckle noise in the reconstructed image of digital holography and its reduction", Opt. Commun. 281, 232-237 (2008). https://doi.org/10.1016/i.optcom.2007.09.030

[29] C. Quan, X. Kang, and C.-J. Tay, "Speckle noise reduction in digital holography by multiple holograms", Opt. Eng. 46, 115801 (2007) https://doi.org/10.1117/1.2802060

[30] L. Rong, W. Xiao, F. Pan, S. Liu, and R. Li, "Speckle noise reduction in digital holography by use of multiple polarization holograms", Chinese Opt. Lett. 8, 653-655 (2010). https://doi.org/10.3788/COL20100807.0653

[31] J. Garcia-Sucerquia, J. Herrera-Ramírez, and R. Castaneda, "Incoherent recovering of the spatial resolution in digital holography", Opt. Commun. 260, 62-67 (2006). https://doi.org/10.1016/j.optcom.2005.10.003

[32] Y. Wang, P. Meng, D. Wang, L. Rong, and S. Panezai, "Speckle noise suppression in digital holography by angular diversity with phase-only spatial light modulator", Opt. Express 21, 19568 (2013). https://doi.org/10.1364/OE.21.019568

[33] T. Nomura, M. Okamura, E. Nitanai, and T. Numata, "Image quality improvement of digital holography by superposition of reconstructed images obtained by multiple wavelengths", Appl. Opt. 47, D38 (2008).

https://doi.org/10.1364/A0.47.000D38

[34] T. Baumbach, E. Kolenovic, V. Kebbel, and W. Jüptner, "Improvement of accuracy in digital holography by use of multiple holograms", Appl. Opt. 45, 6077 (2006). https://doi.org/10.1364/A0.45.006077

[35] J. Herrera-Ramirez, D. A. Hincapie-Zuluaga, and J. Garcia-Sucerquia, "Speckle noise reduction in digital holography by slightly rotating the object", Opt. Eng. 55, 121714 (2016). https://doi.org/10.1117/1.0E.55.12.121714

[36] Y.-X. Ren, R.-D. Lu, and L. Gong, "Tailoring light with a digital micromirror device", Ann. Phys. 527, 447-470 (2015). https://doi.org/10.1002/andp.201500111

[37] D. Dudley, W. M. Duncan, and J. Slaughter, "Emerging digital micromirror device (DMD) applications", Proc. SPIE 4985, 14 (2003). 
https://doi.org/10.1117/12.480761

[38] B. Mills, M. Feinaeugle, C. L. Sones, N. Rizvi, and R. W. Eason, "Sub-micron-scale femtosecond laser ablation using a digital micromirror device", J. Micromechanics Microengineering 23, 35005 (2013). https://doi.org/10.1088/0960-1317/23/3/035005

[39] X.-Y. Ding, Y.-X. Ren, L. Gong, Z.-X. Fang, and R.-D. Lu, "Microscopic lithography with pixelate diffraction of a digital micro-mirror device for micro-lens fabrication", Appl. Opt. 53, 5307 (2014). https://doi.org/10.1364/A0.53.005307

[40] T. Kreis, Handbook of Holographic Interferometry: Optical and Digital Methods, Wiley-VCH Verlag GmbH \& Co., KGaA, Weinheim, (2004).

[41] M. L. Samuels, J. A. Witmer, and A. A. Schaffner, Statistics for the life sciences, Prentice Hall (Pearson), Boston, MA, (2010).

\section{Introduction}

The process of producing a hologram that is digitally recorded and numerically reconstructed, known as Digital holography (DH) [1], has become an opto-digital technique with various applications among which are mechanics, biomedicine, microscopy or spectroscopy [2-11]. This increased use comes from the fact that DH offers the possibility of accessing phase and amplitude of the optical field in a quantitative manner, fact that is a differential feature when compared with other imaging techniques. In spite of its increasing applicability and groups that research around it, DH still suffers from the presence of speckle noise in the final reconstructed image. This is a problem almost ubiquitous to any coherent imaging technique, as the speckle noise comes from the coherent nature of the light necessary in these applications [12]. Consequently, the detrimental effects of speckle in image resolution and misuse of dynamic range of the display devices due to its high contrast are a common concern in coherent imaging applications. In order to overcome this drawback several methods have been proposed [13-35].

Most of the methods proposed to reduce speckle noise in DH can be roughly classified into two different types: the methods coming from a digital processing approach and those coming from an optical approach. On the one hand, the digital processing perspective take advantage of the digital part involved in DH to intervene by using different filtering techniques in either the final reconstructed image [23-25] or the numerical reconstruction step [18-22]. Amongst these digital techniques exist classical filters like mean or median filtering [25], wavelet transform filtering [24] or nonlocal means filtering[23] that operate when the reconstructed image has been obtained. Other approaches intervene in the numerical process of reconstruction modifying the obtained hologram by discrete Fourier filtering [18], using binary masks [19,20], random resampling masks [22] or using a sparsity denoising technique [21]. Although these digital approaches have shown advantages like no need for multiple hologram acquisitions and speed, their results usually compromise the spatial resolution of the final image.

On the other hand, optical methods to reduce speckle in DH are based on acquiring more information of the scene, i.e. more holograms, that preserves object data but with a different distribution in the speckle noise. The concept of devising a synthetic aperture is one of these optical methods [26-28]. This concept is based on acquiring different holograms and subsequently arranging them properly for the numerical reconstruction process. This synthetic enlargement of the aperture, namely the hologram, is effective in reducing speckle size and then improving resolution. Other optical based techniques employ multiple hologram acquisitions as well [29-35], but there is no rearrangement of the holograms to produce a larger aperture. In these cases a superposition in an intensity basis of the individual reconstructions produces the final improvement in the holographic image. This uncorrelated superposition, provided redundant object information, but different speckle noise in each image, produce a speckle contrast in the final result that theoretically follows the curve $1 / \sqrt{N}$ for $N$ superposed speckle patterns [12]. Amongst this type of techniques proposed to produce changes in the speckle distribution in digital holographic images are: using a diffuser in several positions in the object illumination [31], introducing changes in the state of polarization of the object illumination[30], acquiring multiple holograms from different positions [34], slightly rotating the object [35], using multiple wavelengths [33] or rotating the object illumination[29]. All these methods have their own disadvantages like the need of several holograms, long times of acquisition or the use of moving parts, but finally they yield considerable speckle reduction that is their main objective. 
In a similar fashion to this idea of superposition of different speckle patterns, we propose to use a Digital Micromirror Device (DMD) to generate changes in the speckle noise of the reconstructed images of a DH setup. The DMD is a microelectromechanical system with millions of switchable mirrors as pixels [36,37]. Each pixel can be moved to on or off states by inclining them to either $+12^{\circ}$ or $-12^{\circ}$ with respect to the surface normal. The DMD has a high switching speed (up to $9.5 \mathrm{KHz}$ ) and each micromirror has broad spectral response and high reflectivity. Because of these main features, since its invention in 1987 at Texas Instruments, this device has seen a consistent expansion in its applications [37-39]. We introduced the DMD in our holographic setup taking advantage of the mentioned characteristics and that there is no need of any mobile or additional part. Thus, digital holograms are rapidly acquired in correspondence to specific random binary patterns projected by the DMD as object illumination. Several of these holograms are reconstructed and properly superposed to achieve a final image with speckle reduction. The experimental results show that the use of the DMD as object beam modulator is a fast and effective method for speckle noise reduction in $\mathrm{DH}$.

\section{Materials and methods}

Fig. 1 shows a schematic of the digital holographic setup implemented for the proposed method for speckle reduction. In this implementation, light from a solid state laser of $532 \mathrm{~nm}$ wavelength is collimated and divided into the object and reference beams. Using a DMD in the object beam path, specklelike binary patterns are displayed and projected onto the object. The used DMD is part of an evaluation module called DLP ${ }^{\circledR}$ LightCrafter $^{\mathrm{TM}} 6500$ developed by Texas Instruments and third party associates. This DMD is an array of $1920 \times 1080$ squared micromirrors with $7.56 \mu \mathrm{m}$ pixel pitch. The object beam projected and then reflected by the object is aligned with the reference beam to produce off-axis holograms. The holograms are acquired with a Pixelink ${ }^{\circledR}$ CMOS camera with $2592 \times 2048$ pixels and $4.8 \mu \mathrm{m}$ pixel pitch in both directions. The object is located at a distance $z_{0}=90 \mathrm{~cm}$ far from the camera. In this regime the numerical reconstruction of the object field is properly achieved using the Fresnel Transform that in its discrete form is implemented as [40]

$$
\begin{aligned}
U(z)= & \frac{\exp (i k z)}{i \lambda z} \exp \left[\frac{i k}{2 z}\left(\Delta \xi^{2} p^{2}+\Delta \eta^{2} q^{2}\right)\right] \sum_{m=-M / 2 l=-L / 2}^{(M / 2)-1(L / 2)-1} \sum_{h}(m, l) \\
& \exp \left[\frac{i k}{2 z}\left(\Delta x^{2} m^{2}+\Delta y^{2} l^{2}\right)\right] \exp \left[-\frac{i k}{z}(\Delta x m \Delta \xi p+\Delta y l \Delta \eta q)\right]
\end{aligned}
$$

where $U(z)$ represents the reconstructed amplitude on a plane $z$ away from the camera. If $z=z_{0}$ the recovered $U(z)$ equals to the optical wave scattered by the object. $I_{h}(m, l)$ represents the hologram intensity. $\Delta x, \Delta y$ are the pixel sizes at the hologram plane, i.e. the pixel pitches of the CMOS camera, and $\Delta \xi, \Delta \eta$ are the pixel sizes at the reconstruction plane. $m, l$ and $p, q$ are integer counters in the hologram and reconstruction planes, respectively. $k=2 \pi / \lambda$ is the wavenumber with the illuminating wavelength $\lambda$

In order to carry out our proposal to reduce speckle noise, random binary patterns are displayed by the DMD and then projected onto the object. Fig. 2 shows examples of these used binary patterns, so as to change the speckle distribution in our final reconstructed image. Following the analogy of the patterns of the figure with what could be called binary speckle patterns, we use the size of speckle, or grain size (GS), as parameter to describe and generate different kinds of these patterns. Thus, several holograms are recorded each with a different random pattern in the object illumination. These holograms are individually reconstructed using equation (1) keeping the same geometrical parameters in the calculations for all of them. The different object illuminations generate changes in the speckle noise of the reconstructed images in a way that when superposed in an intensity basis the final speckle noise is reduced. This numerical superposition is represented by:

$$
S_{N}=\sum_{n=1}^{N} I_{n}=\sum_{n=1}^{N}\left|U_{n}(z)\right|^{2},
$$

where $N$ is the number of superposed holographic reconstructions with different speckle noise distribution. 


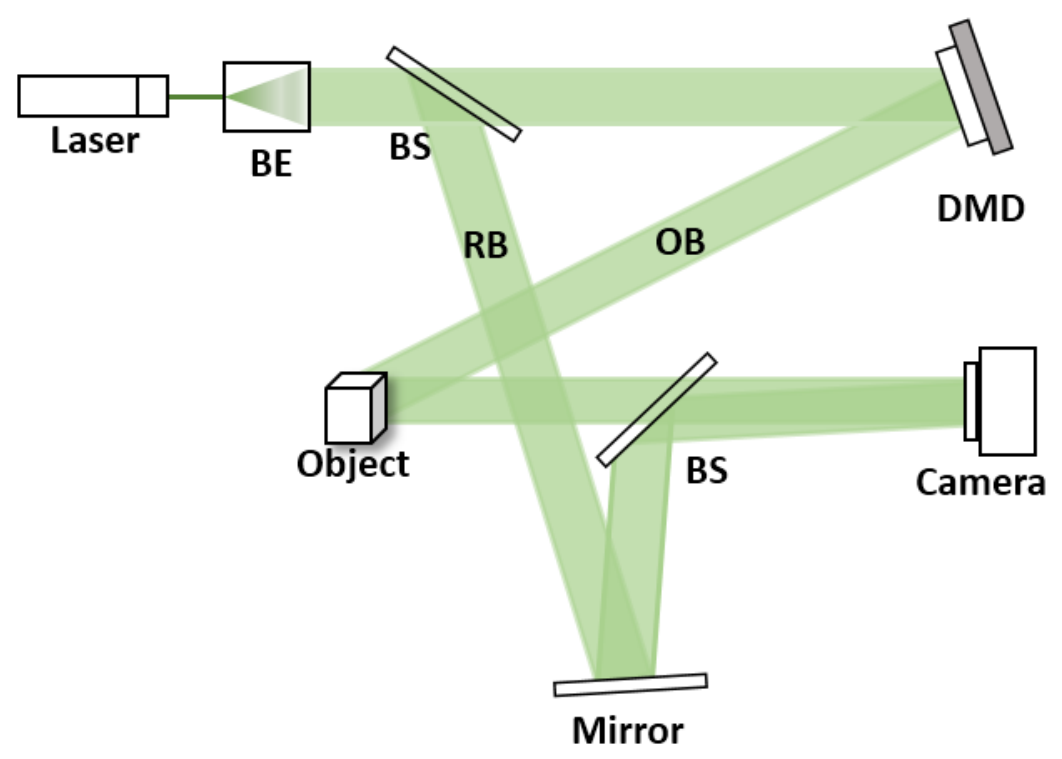

Fig. 1. Experimental setup for off-axis Digital holography using a DMD to modulate object illumination. BE: Beam Expander; BS: Beam Splitter; DMD: Digital Micromirror Device; OB: Object Beam; RB: Reference Beam.

To quantitatively describe a speckle pattern the most used parameter is the speckle index or speckle contrast [12]. We use this speckle contrast descriptor to assess the speckle reduction produced by our method: the lower the value, the better the result in reduction of speckle noise. The speckle contrast is calculated as follows:

$$
C=\frac{\sigma_{I}}{\langle I\rangle}
$$

where $\sigma_{I}$ and $\langle I\rangle$ stand for the standard deviation and mean values of the speckle intensity, respectively.

In the case of totally uncorrelated speckle patterns, the contrast of the superposition of $N$ of such patterns follows a $1 / \sqrt{N}$ behavior [12].
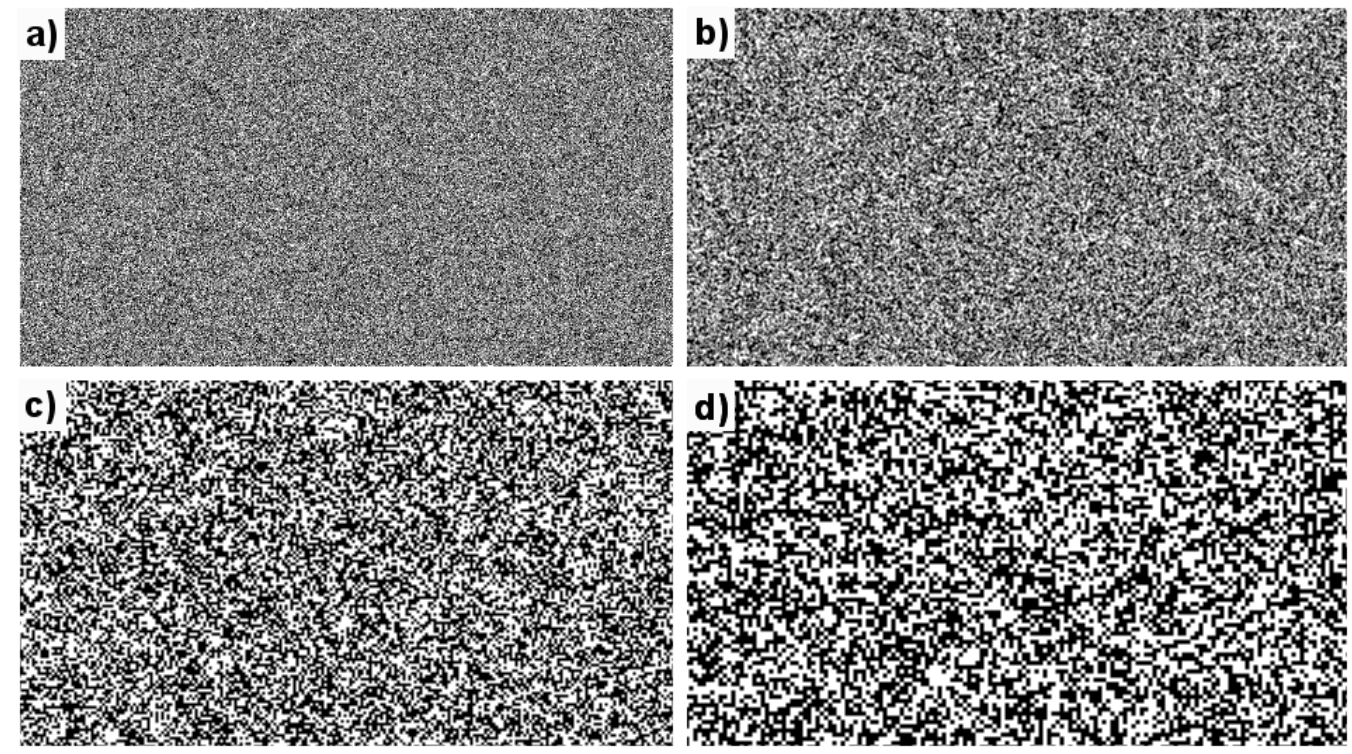

Fig. 2. Speckle-like binary patterns with different grain size (GS) used to be projected as object illumination. a) GS of $1 \times 1$ pixels; b) GS of $4 \times 4$ pixels; c) GS of $8 \times 8$ pixels and d) GS of $12 \times 12$ pixels. 

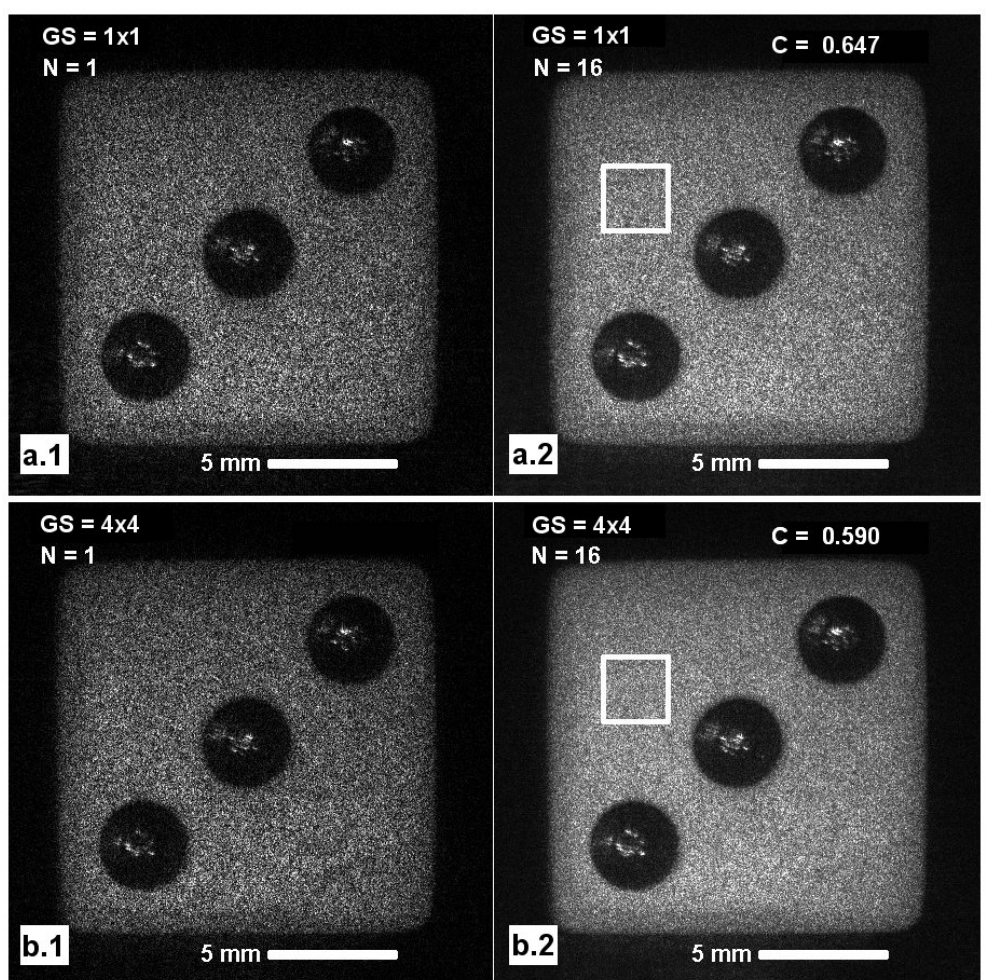

b.1

$5 \mathrm{~mm}$

GS $=8 \times 8 \quad C=0.556$

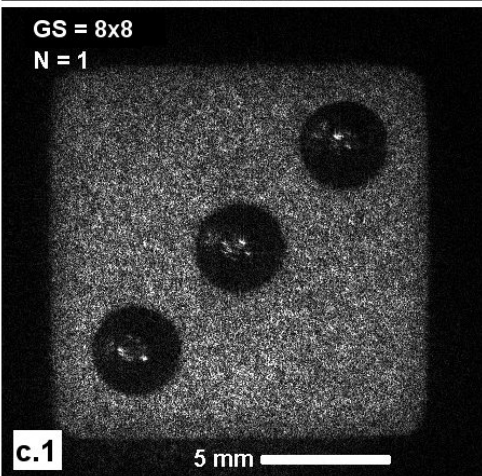

$N=16$

$c=0.556$

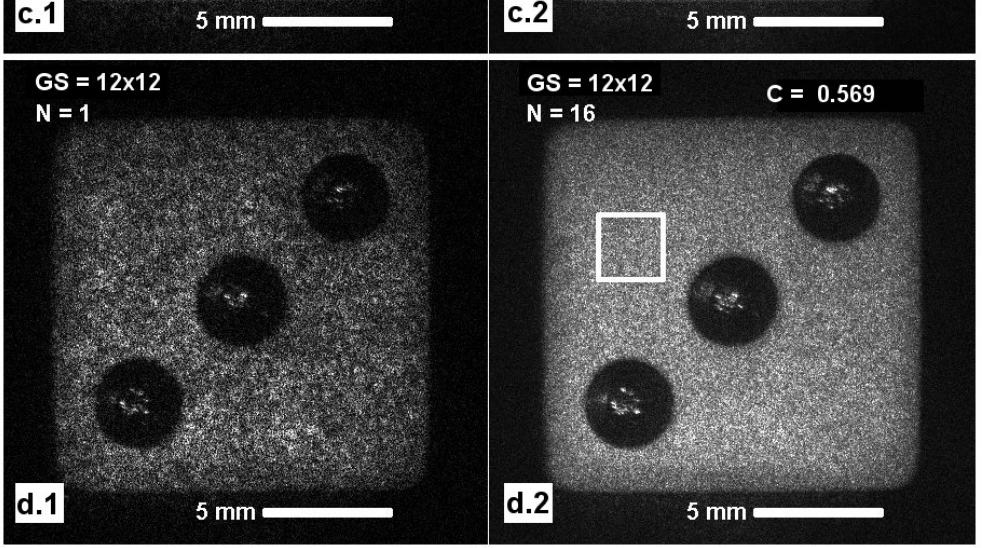

Fig. 3. Experimental results of reduction of speckle contrast achieved by implementing the proposed methodology. Each row contains the results when using speckle-like patterns with the same grain size (GS) in the object illumination: row (a), GS = $1 \times 1$; row (b), GS $=4 \times 4$; row (c), GS $=8 \times 8$; row (d), GS $=12 \times 12$. Each column contains the results when superposing different number of reconstructed images $(N)$ in an intensity basis: column (1), $N=1$; column (2), $N=16$. 


\section{Experimental results in speckle reduction}

Fig. 3 shows the experimental results in the holographic reconstructions when applying the proposed methodology. The images in this figure are arranged by number of superposed images $(N)$ and GS used to generate the patterns in object illumination. Images in the same column have the same $N: N=1$ for column 1 , and $N=16$ for column 2 . Images in the same row have the same GS: GS $=1 \times 1$ for row (a); $\mathrm{GS}=4 \times 4$ for row (b); GS $=8 \times 8$ for row (c); and GS $=12 \times 12$ for row (d). The images in panels a. 1 and b.1 preserve the typical appearance of digital holographic reconstructions, but the images in panels c. 1 and $\mathrm{d} .1$ begin to present an unevenness in the speckle distribution due to the increasing size of GS in their correspondent object illumination. Despite these differences among the results from a single hologram, the results in column 2 with $N=16$ are similar among them because of the process of superposition of several images of the same nature. This process reinforces the information of the object while reducing random variations like speckle. Thus, from the images in column 2, the efficacy of the method can be visually noted. From a quantitative perspective, the speckle contrast values, calculated from the zone delimited by the white square in the images, show that the reduction of speckle noise is slightly different depending on the GS used in the object illumination. The case of GS $=8 \times 8$ presents a $C=0.556$ which is better than the contrast values in the other images, whereas the poorest reduction in speckle is achieved with a GS $=1 \times 1$ where a $C=0.647$ is obtained. Thus, these results show a decrease of speckle contrast around $40 \%$ for $N=16$.

Fig. 4 shows the evolution of speckle contrast value versus the number of superposed intensity reconstructions when using a GS $=8 \times 8$ and $G S=1 \times 1$. The curves with data from experiments have higher contrast than the theoretical curve shown for comparison. Consequently, the efficacy of the proposed method differs from the ideal behavior needing more number of superposed images to achieve the contrast values theoretically expected. Although, the proposed method is still useful in the practice as is shown by the results in column 2 of Fig. 3. Additionally, this reduction in speckle noise can be achieved in a fast manner, due to the high switching rates of the DMD, and may be used in the improvement of image quality in the study of dynamical scenes.

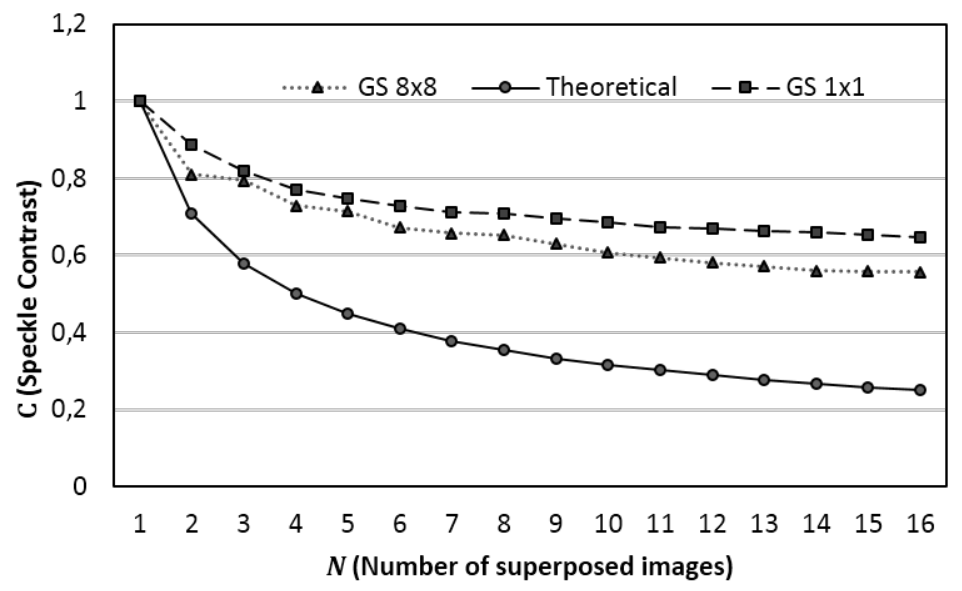

Fig. 4 Speckle contrast curve for the superposition of holographic images generated with a GS $=8 \times 8$ in the object illumination.

Comparison with the theoretical curve $(1 / \sqrt{N})$ for totally uncorrelated speckle patterns.

The difference from the ideal behavior presented by the experimental data in Fig. 4 is explained by the correlation between the speckle patterns in the individual images to be superposed for the final reconstruction. We calculated the correlation coefficient $(\gamma)$ [41] with the intention of evaluating the existing correlation between speckle patterns and so assessing the capacity of the proposed method to reduce this correlation and, in consequence, the speckle noise. The correlation coefficient can be calculated by applying:

$$
\gamma_{t, s}=\frac{\sum_{p}^{M} \sum_{q}^{L}\left[I_{t}(p, q)-\left\langle I_{t}\right\rangle\right]\left[I_{s}(p, q)-\left\langle I_{s}\right\rangle\right]}{\sqrt{\sum_{p}^{M} \sum_{q}^{L}\left[I_{t}(p, q)-\left\langle I_{t}\right\rangle\right]^{2} \cdot \sum_{p}^{M} \sum_{q}^{L}\left[I_{s}(p, q)-\left\langle I_{s}\right\rangle\right]^{2}}},
$$

where $t$ and $s$ stand for each compared image. 
Fig. 5 shows the correlation coefficient between the speckle patterns of two holographic reconstructions while changing the GS values in the object illumination. As mentioned, this coefficient $\gamma$ is related with the capacity of speckle reduction. The ideal curve and highest speckle reduction is obtained if the speckle patterns are totally uncorrelated, i.e. $\gamma=0$. So, as it can be concluded from Fig. 5, the projected patterns by the DMD generate speckle in the reconstructed holograms still partially correlated, and not ideal results are obtained.

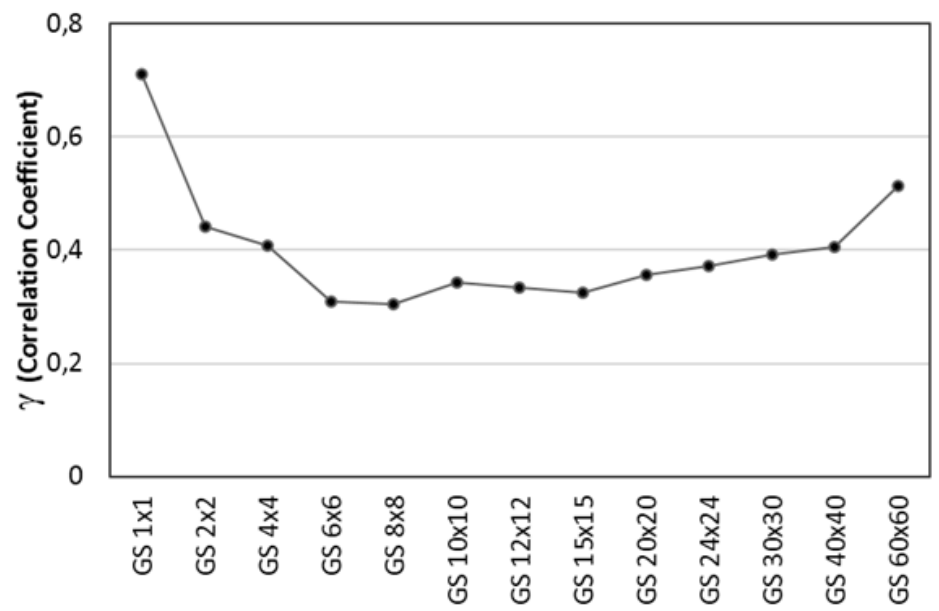

Fig. 5 Correlation coefficient for two reconstructions with a determined GS value in the patterns used for object illumination.

On the other hand, the values in Fig. 5 agree with the results in Fig. 3 showing that the best results in the superposition, using our approach, should be attained with reconstructions using a GS $=8 \times 8$, whilst the results using GS $=1 \times 1$ should perform worst. This figure also suggest that values around GS $=8 \times 8$ have similar performance, but for GS values over $20 \times 20$ this performance decrease again. Fig. 6 presents the result of reconstructions with number of images superposed $N=1$ and $N=16$ when using a value of $\mathrm{GS}=40 \times 40$. It can be observed from the Fig. 6 a that the GS value in the patterns of object illumination also produces spatial variations of the intensity on a larger scale than the scale of the speckle noise of the image. This is expected because of the binary random illumination and was already becoming visible in the images of panels c.1 and d.1 of Fig. 3. These spatial variations are reduced with the process of superposition of images as it is demonstrated in panels c. 2 and d.2 of Fig. 3. But, for high GS values the reduction through superposition of images is not as efficient as in the speckle noise scale, as it is shown in Fig. 6b. Even more, when comparing the speckle contrast values for GS $=1 \times 1$ and GS $=40 \times 40$ being $C=$ 0.647 and $C=0.696$, respectively, they do not agree with what should be expected from the corresponding values of $\gamma$ in Fig. 5. Therefore, high GS values have a double detrimental effect on the quality of the final image reconstruction because: firstly, it produces intensity fluctuations on a larger scale than the speckle size scale; and secondly, it has a decrease on speckle reduction capacity that do not agree with its correspondent correlation value when comparing it with the correlation of low GS values like $1 \times 1$ or $4 \times 4$.

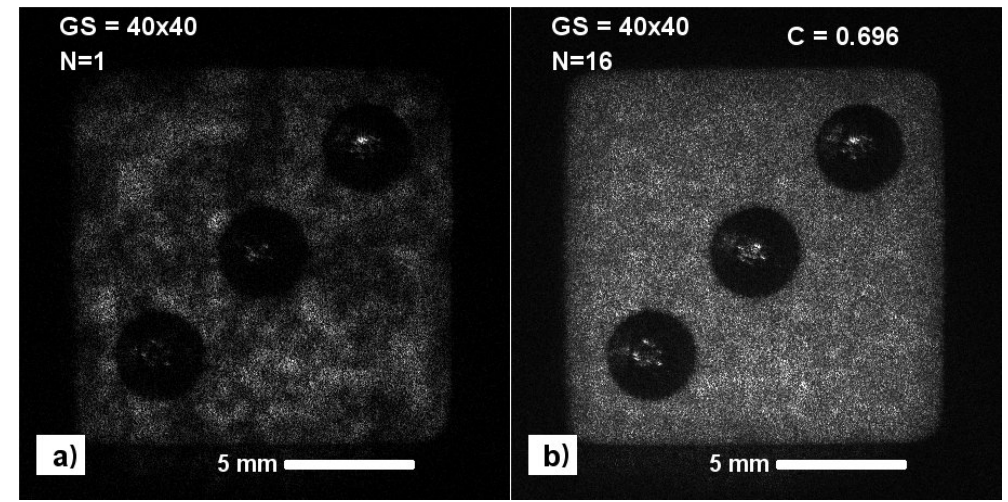

Fig. 6 Results of holographic reconstruction when using GS $=40 \times 40$ in the object illumination. a) $N=1$ superposed images. b) $N=$ 16 superposed images. 


\section{Conclusions}

The proposed method offers an effective alternative to reduce the speckle noise in the numerical reconstruction of digital holograms. Our approach used an easy to implement approach by projecting random binary speckle-like patterns as object illumination. We evaluated the performance of the approach when changing the size of the speckle unit (grain size, GS) in the projected patterns. The evaluation of the speckle contrast descriptor in the reconstructed images jointly with the assessment of the correlation coefficient between speckle patterns in the reconstructions showed that the results obtained with the proposed method differs from the ideal expected values that applies for totally uncorrelated speckle patterns. Despite this undesirable behavior, this approach has worth in the practice because of the almost negligible complexity added by the DMD to the experimental setup and the high rates achievable in the holograms acquisition. These features make this approach to speckle control effective and with possibilities to be used in the study of static as well as dynamical scenes.

The next step in our work is to search for methodologies using the DMD that provide larger decorrelation in the speckle patterns of the reconstructed individual images while still keeping the experimental setup as simple as possible.

\section{Acknowledgements}

The authors acknowledge the support provided by the Instituto Tecnológico Metropolitano (ITM) under the Projects No. P14217 and No. P15108. This work was partially presented in the IX Reunión Iberoamericana de Óptica y XII Reunión Iberoamericana de Óptica, Láseres y Aplicaciones (RIA0/OPTILAS), Pucón, Chile. 